\title{
Risk factors of febrile urinary tract infection in children
}

\begin{abstract}
Background: Urinary tract infection (UTI) implies invasion of the urinary tract by pathogens, which may involve the upper or lower urinary tract depending on the infection in the kidney or bladder and urethra. UTI are the third most common bacterial infection in children in developing countries. The risk factors that predisposes to UTI are diverse and should be evaluated during diagnosis.
\end{abstract}

Objective: To estimate risk factors of febrile UTI patient in children.

Study design: In this case control study, a total of 50 cases and 50 controls were enrolled purposively and conveniently and was carried out in the Department of Pediatric Nephrology and Department of Pediatrics, of Comilla Medical College Hospital, Comilla, Bangladesh between February 2013 to December 2013.

Methods: Cases were selected following clinically suspected by fever, dysuria, urgency, frequency, incontinence, tender renal angle and on urine analysis having $>10$ pus cells/HPF. Then they were confirmed by urine culture whose urine growth were $>105$ colony forming unit per ml. Controls were selected from the patients admitted for other diseases in the Pediatrics ward. Cases and controls were searched for risk factors of urinary tract infection. Data were collected by a preformed structured questionnaire.

Results: Study showed that female sex $(\mathrm{P}<0.05)$, constipation $(\mathrm{P}<0.001)$, not taking anti-helmintic $(\mathrm{P}<0.001)$, lack of toilet training $(\mathrm{P}<0.001)$ and inadequate water intake $(\mathrm{P}<0.01)$ were significant risk factors for urinary tract infection in children and there was no relationship between school going and UTI.

Conclusion: Female sex, constipation, not taking anti-helmintic, lack of toilet training and inadequate water intake are significant risk factors for febrile urinary tract infection in children.

\author{
Volume 2 Issue 5 - 2015
}

\author{
Hossain MA,' Akhter R,' Mannan KA,' \\ Ahmed MS, ${ }^{2}$ Deb KP,' Mostafa G,' Islam \\ AFMA $^{3}$ \\ 'Department of Pediatric Nephrology, Comilla Medical College, \\ Bangladesh \\ ${ }^{2}$ Department of Medicine, Comilla Medical College, Bangladesh \\ ${ }^{3} \mathrm{DCH}$ student, Comilla Medical College, Bangladesh
}

\section{Correspondence: Azizul Hossain, Department of Pediatric Nephrology, Comilla Medical College, Bangladesh, Tel} 0I7IIIII299,Email tasbiul@yahoo.com

Received: July 16, 2015 | Published: November 18,2015

\section{Introduction}

Urinary tract infection (UTI) implies invasion of the urinary tract by pathogens, which may involve the upper or lower urinary tract depending on the infection in the kidney or bladder and urethra. UTI constitutes a common cause of morbidity in association with abnormalities of the urinary tract; resulted in long term complications, including hypertension and chronic renal failure. Prompt detection and treatment of UTI is of the utmost importance. ${ }^{1}$ UTI may be defined by clinical presentation of UTI together with the presence of single pathogenic organism in urinary tract with growth of which is more than $105 \mathrm{cfu}$ per ml. ${ }^{1}$ It is the third most common bacterial infection in children in developing countries after those of the gastrointestinal and respiratory tract. Because of nonspecific signs and vague symptoms in very young children, they remain unrecognized and therefore precise data on incidence and prevalence of UTI are not available. The commonest age for the occurrence of the first symptomatic UTI is the first year of life and male affected more than female. ${ }^{1}$ During infancy, the risk of developing UTI is equal in boys and girls and thereafter higher in girls. ${ }^{2}$

About 90 percent of the first symptomatic UTI and 70 percent of recurrent infections are due to E. coli. Proteus species may be causative in about a third of boys with acute cystitis. Other organisms including Klebsiella, staphylococcus epidermidis and streptococcus faecalis may occasionally be responsible. Pathogens of low virulence and fungi may be causative agent in patients who are immunocompromised. Candida albicans infections are particularly seen in preterm babies. $^{2}$

UTI may be suspected based on symptoms and findings on urine analysis or both. A urine culture is necessary for confirmation and appropriate therapy. ${ }^{1}$ There are several methods to obtain a urine sample. Some are more accurate than others. In toilet-trained children, a midstream urine sample usually is satisfactory; the introitus should be cleaned before obtaining the specimen. In uncircumcised boys, the prepuce must be retracted; if the prepuce is not retractable; avoided sample may be unreliable and contaminated with skin flora. In children who are not toilet-trained, a catheter sample is better but too invasive. ${ }^{1}$

The risk factors of UTI are numerous; they may be age, gender, constipation, lack of circumcision, inadequate water intake, not taking anti-helmintic and lack of toilet training. As male are more likely to be born with structural abnormalities of the urinary tract, UTI is more common in their first six months of life. ${ }^{3}$ Few infections are encountered in boys in the age range of 1-5years. In pre-school years symptomatic infections occur 10 to 20times more frequently in girls than in boys. ${ }^{4}$ Constipation is a common problem of children worldwide. Estimates of the prevalence ${ }^{5,6}$ rate of functional constipation in the pediatric population have varied from $4 \%$ to $37 \%$. Enterobius vermicularis is one of the most prevalent worms found in children worldwide. Children, particularly when there are heavy worm burdens, neurological symptoms such as nervousness, restlessness, 
irritability and distraction may occur and these may influence on child growth ${ }^{7}$ and may occur urinary tract infections. ${ }^{8}$ Uncircumcised male infants appear to be at increased risk of UTIs. The periurethral area was found to be more frequently and more heavily colonized with uropathogens, especially Escherichia coli, in uncircumcised infants than in circumcised infants. ${ }^{9}$ Winberg et al., ${ }^{9}$ offered an explanation for the high incidence of UTIs in uncircumcised male infants in an intriguing article.

\section{Rationale}

Urinary tract infection is a great morbidity in children. It encompasses renal damage as well as mortality of children. Many risk factors are responsible for initial urinary tract infection and recurrent urinary tract infection. But there is scanty data in Bangladesh to estimate the risk factors of febrile urinary tract infection by case control study. The goal of our study was to shed some light in some risk factors which are common in urinary tract infection in children. So that it can create public awareness to prevent UTI and improve the well being of children.

\section{Patients and methods}

The objectives of this study were to identify the risk factors of febrile urinary tract infection in children. This was a case control study carried out in the Department of Pediatric Nephrology and Department of Pediatrics, Comilla Medical College, Bangladesh from February 2013 to December 2013.

\section{Studied population}

All UTI patients of children were included in this study with the following inclusion and exclusion criteria.

\section{Inclusion criteria}

Children who were between 5years to 12 years old, can give history of fever, abdominal pain, dysuria, urgency, frequency of micturition and able to give urine sample aseptically by mid stream urine and cleaning the external urethral orifice were included in this study.

\section{Exclusion criteria}

Children whose history were difficult to take from the parents, unable to give urine sample and having other cause of fever like viral respiratory tract infection, other cause of abdominal pain like acute appendicitis or gastro enteritis and who had congenital anomalies of urinary tract detected by ultra sonogram were excluded from the study.

\section{Sampling method and sample size}

Purposive sampling method was applied and conveniently 50 samples were taken as cases.

\section{Controls}

50 Controls were selected from the Department of Pediatrics who had no symptoms of urinary tract infection and excluded by laboratory investigations.

\section{Procedure}

Immediately after admission, detailed history and physical examination of the patient was done. Cases were selected from Pediatric Nephrology ward when clinically suspected as febrile UTI by fever, dysuria, urgency, frequency, abdominal pain, malodour urine, incontinence, tender suprapubic region, tender renal angle and on urine analysis having $>10$ pus cells/HPF2. Then they were confirmed by urine culture whose urine growth were $>105$ colony forming unit per ml1. Negative culture patients were excluded from the study. Controls were selected from the patients admitted for other diseases in the pediatrics ward. Cases and controls were searched for risk factors of urinary tract infection. Data were collected by a preformed structured questionnaire and were analyzed using computer software and SPSS. Significance of difference was calculated using, chi-square $(x 2)$ test. Multiple regression analysis was also calculated out.

\section{Flow chart}

Initially 500 patients were included in this study based on clinical features $\longrightarrow$ Among them 200 were found positive urine analysis from those patient 50 patients were found positive urine $\longrightarrow$ culture this 50 patients were searched for risk factors of UTI and were compared with 50 controls by statistical analysis.

\section{Ethical issues}

Ethical issues were addressed duly by taking permission of parents and approval from ethical committee of Comilla Medical College, Bangladesh. Total 50 cases and 50 controls were included in this study. The mean age of cases and control were 7.4years and 7.8years respectively. Regarding sex 22 were male and 28 were female among cases and 34 were male and 16 were female among controls. Among the cases $100 \%$ were having clinical features of UTI, positive urine analysis and positive urine culture. None of the cases and controls had congenital anomalies of urinary tract detected by ultra sonogram (Table 1-3).

Table I Baseline characteristics of cases \& controls

\begin{tabular}{|c|c|c|c|c|}
\hline & & Cases & Controls & $P$ value \\
\hline 1 & Total number & 50 & 50 & - \\
\hline 2 & Age( years) & 7.5 & 7.7 & $>.05$ \\
\hline 4 & \multirow[b]{2}{*}{ Female } & 22 & 34 & - \\
\hline & & 28 & 16 & - \\
\hline 5 & Clinical features present (\%) & 100 & - & - \\
\hline 5 & Urine R/M/E (\%) & 100 & - & - \\
\hline 6 & Urine culture (\%) & 100 & - & - \\
\hline 7 & USG findings of urinary tract & nil & nil & \\
\hline
\end{tabular}


Table 2 Effect of different risk factors independently associated with UTI

\begin{tabular}{lllll}
\hline Factors & Case & Controls & $\mathbf{x}^{2}$ & P value \\
\hline Female sex & 28 & 16 & 5.8 & $<0.05$ \\
Constipation & 32 & 15 & 11.6 & $<0.00 \mathrm{I}$ \\
Lack of circumcision & 7 & 12 & 4.9 & $<0.05$ \\
Not taking anthelmintic & 15 & 28 & 6.9 & $<0.01$ \\
Lack of toilet training & 10 & 14 & 23.3 & $<0.001$ \\
Inadequate water intake & 5 & 17 & 8.3 & $<0.01$ \\
Effect of School going & 37 & 37 & 0 & $>.5$ \\
\hline
\end{tabular}

Table 3 Multiple logistic regression analysis results showing factors associated with UTI infection in children

\begin{tabular}{lll}
\hline Factors & OR $(\mathbf{9 5} \% \mathbf{C I})$ & P-value \\
\hline Female sex & I.5(I.33-I.58) & $<0.05$ \\
Constipation & $5(2.89-9.57)$ & $<0.05$ \\
Lack of circumcision & I.2(I.I0-3.65) & $<0.05$ \\
Inadequate water intake & I.3(I.23-4.4I) & $<0.05$ \\
Not taking anthelmintic & I.5(I.32-3.67) & $<0.05$ \\
School going & I $(0.40-2.44)$ & $\mathrm{I}$ \\
Lack of toilet training & $\mathrm{I} .6(\mathrm{I} .15-2.93)$ & $<0.00 \mathrm{I}$
\end{tabular}

\section{Interpretation}

Female sex, constipation, lack of circumcision, not taking anthelmintic, lack of toilet training, inadequate water intake had significant effect independently on UTI but school going had no effect on UTI.

\section{Interpretation}

Multiple Logistic Regression analysis results showed multiple independent variables like female sex, constipation, lack of circumcision, inadequate water intake, not taking anti-helmintic and lack of toilet training were significant risk factors for dependent variable UTI but school going had no effect on UTI.

\section{Discussion}

Urinary tract infections (UTIs) are more common among girls than boys. The predominant UTI risk factors in young girls are anatomic and physiologic factors. The short urethra of female permits easy entry of bacteria into the bladder and causes UTI. In our study we have found female baby are more prone to developed UTI than male baby $(\mathrm{p}<.05)$. Similar results are found by Foxman. ${ }^{10}$ Harrington and Hooton also supported our results. ${ }^{11}$ This finding also supported by Nader et al., ${ }^{12}$ Steven. ${ }^{13}$

It has been shown that constipation with a dilated rectum causes the same pattern of voiding dysfunction as that encountered in children with persistence of an unstable bladder. Effective treatment of the constipation results in normalization of bladder function and cessation of UTIs. ${ }^{14}$ In our study we have found constipation is a risk factor to developed UTI $(p<0.001)$. A relationship between constipation and UTIs is well described by O'Regan et al. ${ }^{15}$ Farid Imanzadeh and AliAkbar found similar result. ${ }^{16}$

In one study, appropriate hygiene decreased significantly the incidence of phimosis, adhesions, and inflammation. ${ }^{17} \mathrm{We}$ have found UTI are more common in male children who did not get circumcision $(\mathrm{p}<0.05)$. Herzog suggested in a case control study that there is a correlation between urinary tract infections and circumcision. ${ }^{18}$ This also supported by many other studies. ${ }^{19,20}$

Enterobiasis is most common in children between 5years and $10 y e a r s$ of age. Serious complications like urinary tract infection may occur due to pin worm. Regular anti-helmintic taking may reduce the prevalence of urinary tract infection. We have found taking antihelmintic reduce the infection of UTI $(p<0.01)$. This is supported by study conducted by Ok UZ et al., ${ }^{20}$ Gokalp et al. ${ }^{21}$ Effect of lack of toilet training is a significant risk factor of UTI $(\mathrm{P}<0.001)$. We can conclude that lack of toilet training is extremely high relationship with UTI. This was supported by study conducted by Chen JJ et al. ${ }^{22}$

Bacterial eradication from the urinary tract is partially dependent on urine flow and voiding frequency. Therefore, it seems logical to postulate a connection between fluid intake and the risk of urinary tract infections (UTIs). In our study we found inadequate water intake predisposes to developed urinary tract infection $(\mathrm{p}<.01)$. Denman SJ \& Burton JR suggested that mild dehydration is a risk factor of urinary tract infection. ${ }^{23}$

\section{Limitation of this study}

Small sample size is one of the limitations of the study. Due to lack of kits we did not perform nitrate Patient did not co operate to do MCU to exclude VUR.

\section{Conclusion}

Female sex, constipation, not taking anti-helmintic, lack of toilet training and inadequate water intake are significant risk factors for febrile urinary tract infection in children.

\section{Acknowledgements}

None.

\section{Conflict of interest}

Author declares that there is no conflict of interest.

\section{References}

1. Jack S Eider. Urinary tract infection. In Nelsion Textbook of Pediatrics. 19th ed. Saunders Publishers; 2012. p. 1829-1834.

2. Srivasta, Bagga A. Pediatric Nephrology. 5th ed. New Delhi: Jaypee Brothers, Medical Publishers; 2011. p. 235-250.

3. Shaw KN, McGowan KL, Gorliek MH, et al. Screening for urinary tract infection in Infants in the Emergency Department: Which Test Is Best? Pediatrics. 1998;101(6):E1.

4. Schlager TA. Urinary tract infections in infants and children. Infect Dis Clin North Am. 2003;17(2):353-365.

5. Van der Wal MF, Benninga MA, Hirasing RA. The prevalence of encopresis in a multicultural population. J Pediatr Gastroenterol Nutr. 2005;40(3):345-348.

6. de Araújo Sant, Anna AM, Calçado AC. Constipation in school aged children at public schools in Rio de Janeiro, Brazil. J Pediatr Gastroenterol Nutr. 1999;29(2):190-193.

7. Kang S, Jeon HK, Eom KS, et al. Egg positive rate of Enterobius vermicularis among preschool children in Cheongju, Chungcheongbukdo, Korea. Korean J Parasitol. 2006;44(3):247-249. 
8. Song HJ, Cho CH, Kim JS, et al. Prevalence and risk factors for enterobiasis among preschool children in a metropolitan city in Korea. Parasitol Res. 2003;91(1):46-50.

9. Winberg J, Bollgren I, Gothefors L, et al. The prepuce: a mistake of nature? Lancet. 1989;(8638):598-599.

10. Foxman B. Epidemiology of urinary tract infections: incidence, morbidity, and economic costs. Dis Mon. 2003;49(2):53-70.

11. Harrington RD, Hooton TM. Urinary tract infection risk factors and gender. J Gend Specif Med. 2000;3(8):27-34.

12. Shaikh N, Morone NE, Bost JE, et al. Prevalence of Urinary Tract Infection in Childhood: A Meta Analysis. Pediatr Infect Dis J. 2008;27(4):302-308.

13. Chang SL, Shortliffe LD. Pediatric Urinary Tract Infections. Pediatr Clin N Am. 2006;53(3):379-400.

14. Loening Baucke V. Urinary incontinence and urinary tract infection and their resolution with treatment of chronic constipation of childhood. Pediatrics. 1997;100(2 Pt 1):228-232.

15. O’Regan S, Yazbeck S, Schick E. Constipation, bladder instability, urinary tract infection syndrome. Clin Nephrol. 1985;23(3):152-154.
16. Farid Imanzadeh, Ali-Akbar Sayyari, Mostafa Sharifian, et al. Study of factors affecting resolution of urinary tract infection following treatment of constipation in Iranian children who visited a tertiary referral hospital, Przegląd. Gastroenterologiczny. 2012;7(2):78-80.

17. Krueger H, Osborn L. Effects of hygiene among the uncircumcised. $J$ Fam Pract. 1986;22(4):353-355.

18. Herzog LW. Urinary tract infections and circumcision: A case control study. Am J Dis Child. 1989;143(3):348-350.

19. Shaikh N, Morone NE, Lopez J, et al. Does This Child Have a Urinary Tract Infection? JAMA. 2007;298(24):2895-2904.

20. Ok UZ, Ertan P, Limoncu E, et al. Relationship between pinworm and urinary tract infections in young girls. APMIS. 1999;107(5):474-476.

21. Gokalp A, Gultekin E, Kiersi M, et al. Relation between Enterobius vermicularies infestation and dysuria, noctunuria, enuresisnocturuia and bacteruria in primary school girls. Indian Pediatr. 1991;28(8):948-950.

22. Chen JJ, Ahn HJ. Is age at toilet training associated with the presence of vesicoureteral reflux or the occurrence of urinary tract infection? $J$ Urol. 2009;182(1):268-271.

23. Denman SJ, Burton JR. Fluid intake and urinary tract infection in the elderly. JAMA. 1992;267(16):2245-2249. 